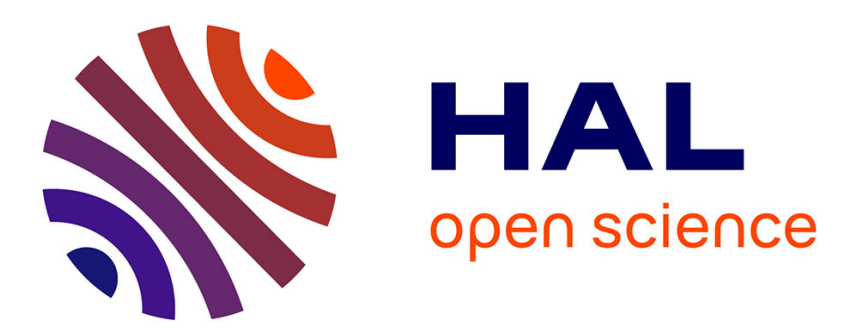

\title{
Les effets différenciés de la réforme de la PAC sur les revenus des agriculteurs
}

\author{
Alain Blogowski, Philippe Boyer
}

\section{To cite this version:}

Alain Blogowski, Philippe Boyer. Les effets différenciés de la réforme de la PAC sur les revenus des agriculteurs. Economie Rurale, 1994, 10.3406/ecoru.1994.4627 . hal-02964267

\section{HAL Id: hal-02964267 \\ https://hal.science/hal-02964267}

Submitted on 12 Oct 2020

HAL is a multi-disciplinary open access archive for the deposit and dissemination of scientific research documents, whether they are published or not. The documents may come from teaching and research institutions in France or abroad, or from public or private research centers.
L'archive ouverte pluridisciplinaire HAL, est destinée au dépôt et à la diffusion de documents scientifiques de niveau recherche, publiés ou non, émanant des établissements d'enseignement et de recherche français ou étrangers, des laboratoires publics ou privés. 


\title{
Les effets différenciés de la réforme de la PAC sur les revenus des
} agriculteurs

\author{
M. Alain Blogowski, Mr Philippe Boyer
}

\section{Citer ce document / Cite this document :}

Blogowski Alain, Boyer Philippe. Les effets différenciés de la réforme de la PAC sur les revenus des agriculteurs. In: Économie rurale. $N^{\circ} 220-221,1994$. Les revenus agricoles. Session de printemps 1993, 13 et 14 mai, au IAM de Montpellier, organisée par Jean-Pierre Butault, Bernard Delord et Patrick Rio, chercheurs au Département Economie et Sociologie Rurales de I'INRA. pp. 124-130;

doi : https://doi.org/10.3406/ecoru.1994.4627

https://www.persee.fr/doc/ecoru_0013-0559_1994_num_220_1_4627

Fichier pdf généré le 08/05/2018 


\begin{abstract}
The effects of c.a.p. reform on farmer's incomes

In France, the CAP reform will involve an average increase of Gross Operating Surplus equal to 1. 7 per cent from 1991 to 1996, and 3 per cent for the Net Income. Direct subsidies will increase to 34 milliards of Francs and reach about 38 per cent of the Gross Operating Surplus. The farm specialisations differ in the growth of the direct subsidies share in the Gross Operating Surplus from 1991 to 1996 : 4 p.c. to 85 p.c.for cereal-specialised farming, 40 p.c. to 71 p.c. for bovine-meat breeding. The distribution of the incomes will become narrower because of the diminution of cereal farms incomes (-19 p.c.) and the increase of cattle farm incomes (from 9 p.c. to 18 p.c, according to specialisation).
\end{abstract}

\title{
Résumé
}

Les changements introduits dans le mode de gestion des prix et des revenus agricoles dans la Communauté conduiraient en 1996 (réforme accomplie) à une augmentation moyenne de 1,7\% de l'Excédent Brut d'Exploitation (par rapport à l'année 1991). Les aides directes augmenteraient de 34 milliards de francs et représenteraient en période de pleine application de la réforme près de $38 \%$ de l'Excédent Brut d'Exploitation de la branche, contre $10 \%$ en 1991. On constate en outre :

-un fort accroissement du poids des aides directes dans la formation du revenu : de 1991 à 1996, elles passent de $4 \%$ à $85 \%$ de l'EBE pour l'OTEX "céréales" et de 40 à $71 \%$ pour la viande bovine;

-un resserrement de l'éventail des revenus, lié à la baisse des revenus céréaliers (- 19\%), et à l'amélioration de la situation des exploitations d'élevage (de $+9 \%$ à $+18 \%$ selon les orientations considérées). 


\title{
LES EFFETS DIFFERENCIES DE LA REFORME DE LA PAC SUR LES REVENUS DES AGRICULTEURS
}

\author{
Alain BLOGOWSKI, Philippe BOYER \\ Ministère de l'Agriculture et de la Pêche, Direction des Affaires Financières et Economiques, SDEPE, Bureau \\ Evaluation et Prospective

\section{Résumé :} \\ Les changements introduits dans le mode de gestion des prix et des revenus agricoles dans la Communauté \\ conduiraient en 1996 (réforme accomplie) à une augmentation moyenne de 1,7\% de l'Excédent Brut \\ d'Exploitation (par rapport à l'année 1991). Les aides directes augmenteraient de 34 milliards de francs et \\ représenteraient en période de pleine application de la réforme près de $38 \%$ de l'Excédent Brut d'Exploitation \\ de la branche, contre $10 \%$ en 1991 . On constate en outre : \\ - un fort accroissement du poids des aides directes dans la formation du revenu : de 1991 à 1996, elles passent \\ de $4 \%$ à $85 \%$ de l'EBE pour l'OTEX "céréales" et de 40 à $71 \%$ pour la viande bovine; \\ - un resserrement de l'éventail des revenus, lié à la baisse des revenus céréaliers (- 19\%), et à l'amélioration de \\ la situation des exploitations d'élevage (de $+9 \%$ à $+18 \%$ selon les orientations considérées).

\section{THE EFFECTS OF C.A.P. REFORM ON FARMER'S INCOMES}

\section{Summary :} \\ In France, the CAP reform will involve an average increase of Gross Operating Surplus equal to 1.7 per \\ cent from 1991 to 1996, and 3 per cent for the Net Income. Direct subsidies will increase to 34 milliards of \\ Francs and reach about 38 per cent of the Gross Operating Surplus. The farm specialisations differ in the \\ growth of the direct subsidies share in the Gross Operating Surplus from 1991 to $1996: 4$ p.c. to 85 p.c. for \\ cereal-specialised farming, 40 p.c. to 71 p.c. for bovine-meat breeding. The distribution of the incomes will \\ become narrower because of the diminution of cereal farms incomes $(-19$ p.c.) and the increase of cattle \\ farm incomes (from 9 p.c. to 18 p.c., according to specialisation).
}

La réforme de la Politique Agricole Commune adoptée le 21 mai 1992 a fait l'objet de multiples publications visant à en expliciter les modalités d'application et à en estimer les conséquences.

Les travaux les plus récents visent notamment à évaluer les effets des différentes mesures sur le revenu, d'une part dans une perspective macroéconomique (laquelle s'intéresse également aux effets sur l'équilibre offre-demande et sur les dépenses budgétaires), d'autre part à l'échelle micro-économique (revenu des exploitations). Toutefois, cette dernière approche ne recouvre encore que des études de systèmes de production géographiquement circonscrits, ou des analyses de cas-types, souvent davantage illustratifs d'une thèse que véritablement représentatifs.

Il nous a donc paru nécessaire de conduire une étude micro-économique à caractère exhaustif (ensemble des régions et des orientations de production) pour analyser les effets différenciés de la réforme sur le revenu des agriculteurs, identifier les exploitations dont la pérennité, ou la reproductibilité, pourrait être compromise et évaluer le degré de dépendance des résultats économiques au montant et à la nature des aides.

Pour répondre à ces différents objectifs nous avons utilisé les données du RICA relatives à l'exercice 1991 auxquelles nous appliquons, sans modification des structures existantes, les variations de prix et les montants d'aides compensatoires prévus à l'échéance 1996. Il n'est donc actuellement fait aucune hypothèse d'adaptation des exploitations aux nouveaux dispositifs. Toutefois, dans la dernière partie de cette note, nous présenterons brièvement les premiers résultats obtenus suite à l'introduction de quelques scénarios simples d'adaptation.

Les données qui figurent ci-après sont à considérer comme des ordres de grandeur, et non comme des prévisions. 


\section{LES EFFETS GLOBAUX DE LA REFORME SUR LE REVENU AGRICOLE}

Les conséquences des mesures communautaires et du plan national d'accompagnement sont simulées pour chaque exploitation du RICA. Un programme de calcul détermine les effets de chaque mesure sur les différents postes comptables, à l'exception de ceux non directement modifiés par la nouvelle réglementation ${ }^{1}$. S'agissant d'une approche statique (atemporelle), il n'est fait aucune hypothèse monétaire (inflation) ou démographique (diminution du nombre d'exploitations).

Les effets "mécaniques" de la réforme de la P.A.C. conduiraient en 1996 (réforme accomplie), à une augmentation moyenne du revenu de 3700 francs par exploitation. Cette variation représente une hausse de 1,7\% de l'Excédent Brut d'Exploitation (par rapport à l'année 1991), et de 3\% du Résultat Courant Avant Impôts ${ }^{2}$.

Ce résultat peut se décomposer, sommairement, de la façon suivante :

\section{Budget partiel (millions de Francs) ${ }^{3}$}

\begin{tabular}{|c|c|c|c|}
\hline Baisse de produits & $\begin{array}{l}\text { dont prod. végét. } \\
\text { dont prod. anim. }\end{array}$ & -42200 & $\begin{array}{r}-35200 \\
-7000\end{array}$ \\
\hline Economie de charges & $\begin{array}{l}\text { dont aliments } \\
\text { dt charg. cultur. } \\
\text { dt taxes coresp. } \\
\text { dont TFNB }\end{array}$ & 10100 & $\begin{array}{ll}1600 \\
4100 \\
3300 \\
1100\end{array}$ \\
\hline $\begin{array}{l}\text { Solde hors aides directes } \\
\text { Subventions supprimées } \\
\text { Subv. noua. ou en augm. }\end{array}$ & & $\begin{array}{r}-32200 \\
-700 \\
34800 \\
\end{array}$ & \\
\hline
\end{tabular}

\section{VARIATION DE REVENU :}

+2 milliards de francs

Détail des aides nouvelles et des revalorisations (millions de Francs) :

\begin{tabular}{|l|r|}
\hline Aides au gel des terres & 4300 \\
\hline Aides au maïs fourrage & 3600 \\
\hline Autres aides aux grandes cultures & 22000 \\
\hline Sous-total & 29900 \\
\hline
\end{tabular}

\begin{tabular}{|l|r|}
\hline Aides animales communautaires & 3900 \\
\hline Indemnités compensatrices de handicaps & 200 \\
\hline Primes à l'herbe & 900 \\
\hline Sous-total & 5000 \\
\hline
\end{tabular}

\footnotetext{
1 Prix et volumes des productions hors OCM et par conséquent, valeurs de leurs intrants spécifiques.

2 L'écart entre ces estimations et celles présentées lors de la session de la SFER est essentiellement lié à la revalorisation de l'aide au gel des terres.

3 Valeurs arrondies.
}

Le revenu augmenterait donc de 2 milliards de francs et les aides directes de 34 milliards. En conséquence, ces dernières représenteraient, en fin de période, 38\% de l'Excédent Brut d'Exploitation, contre $10 \%$ en 1991 . Ramenée au résultat courant, cette proportion passerait de 18 à $69 \%$.

Les mesures nationales du plan d'accompagnement s'élèveraient à 2,2 milliards pour l'exercice 1996. En l'absence de telles mesures, l'évolution de l'EBE aurait donc été légèrement négative.

Les pertes de recettes liées à la baisse des prix du lait et de la viande bovine seraient plus que compensées par l'augmentation des aides animales et par les primes au maïs fourrage. Il n'en est, par contre, pas de même pour les baisses de recettes végétales qui restent supérieures aux compensations directes et aux économies réalisées sur les charges culturales.

\section{LES PRINCIPALES ORIENTATIONS DE PRODUCTION - FRANCE ENTIERE}

La hausse de 1,7\% de l'EBE moyen masque, en fait, de profondes disparités. Ainsi, alors que le revenu des céréaliers baisserait d'environ $19 \%$, celui des éleveurs augmenterait de $9 \%$ pour les exploitations laitières, et de $18 \%$ pour celles spécialisées dans la production de "bovins élevage et viande".

\section{II.1 Les grandes cultures}

Les pertes de revenu sont plus prononcées pour les exploitations spécialisées en céréales $(-19 \%)$ que pour les exploitations plus diversifiées (oléagineux, betteraves,...), où l'EBE ne diminuerait "que" de $9 \%$. Le revenu moyen de ces exploitations resterait toutefois élevé : 200000 francs pour les céréaliers et 250000 francs pour l'orientation "céréales et autres grandes cultures". Les économies "mécaniques" réalisées sur les charges variables compensent environ $10 \%$ des pertes de recettes.

\section{II.2 Les élevages bovins}

\section{Bovins lait}

Les primes à l'élevage ( 5700 francs en moyenne par exploitation) ${ }^{4}$ sont inférieures à la perte de produit animal (19 700 F). Toutefois, les aides au mais fourrage (15 300 F), au gel et aux autres cultures (12 600 F) assurent un solde positif qui permet au revenu d'enregistrer une progression de $9 \%$. Les mesures nationales participent pour $28 \%$ à l'augmentation du revenu.

\begin{tabular}{|l|r|}
\hline Aides aux cultures (hors maïs fourrage) & $11500 \mathrm{~F}$ \\
\hline Aides au mais fourrage & $5600 \mathrm{~F}$ \\
\hline $\begin{array}{l}\text { Augmentation des primes animales } \\
\text { communautaires }\end{array}$ & $34000 \mathrm{~F}$ \\
\hline $\begin{array}{l}\text { Augmentation des autres primes } \\
\text { (ISM, prime à l'herbe) }\end{array}$ & $6500 \mathrm{~F}$ \\
\hline
\end{tabular}

$4 \mathrm{Y}$ compris la "prime à lherbe" et les indemnités compensatrices de handicaps naturels. 


\section{Bovins viande}

L'OTEX 42 enregistre la plus forte progression de revenu (18\%). Ces exploitations bénéficient de l'ensemble des mesures prévues par la réforme :

Les mesures nationales contribuent pour $34 \%$ à l'augmentation de l'E.B.E.. Toutefois, malgré cette très forte hausse, l'E.B.E. moyen des éleveurs de viande bovine resterait en 1996 inférieur de $40 \%$ à celui des exploitations orientées vers la production de céréales et d'autres grandes cultures.

\section{Bovins mixte (lait et viande)}

En hausse de $12 \%, l^{\prime}$ EBE de ces exploitations bénéficie, comme précédemment, de l'ensemble des mesures prévues par la réforme. Les pertes de recettes animales ne sont pas intégralement compensées par les aides prévues à cet effet. Toutefois, les 16000 francs, en moyenne par exploitation, liés à l'indemnisation du maïs fourrage concourent à la progression substantielle du revenu de ces éleveurs. Les mesures nationales représentent $19 \%$ de ce gain.

\section{II.3 Ovins et autres herbivores}

Le revenu de ces exploitations progresse en moyenne de $17 \%$ grâce à : des aides directes aux cultures trois fois plus élevées que les pertes de recettes (faibles rendements et indemnisation des céréales intraconsommées) ; des aides aux productions animales qui compensent en grande partie les pertes occasionnées par la baisse des prix, et d'importantes subventions liées au plan d'accompagnement (9 200 F. en moyenne par exploitation, soit $37 \%$ du supplément de revenu).

\section{II.4 Aides directes et revenus}

* Les exploitations de "grandes cultures", qui constituent $20 \%$ des exploitations agricoles du champ d'observation percevraient, en $1996,40 \%$ du total des subventions d'exploitation. Malgré ces 18 milliards d'aides, leur revenu baisserait notablement et la part des aides directes dans la composition de leur résultat oscillerait entre 67 et $85 \%$ selon leur degré de spécialisation céréalière.

\begin{tabular}{|c|c|c|c|c|c|c|c|}
\hline & Céréales & $\begin{array}{l}\text { Aut. gdes } \\
\text { cultures }\end{array}$ & Lait & $\begin{array}{l}\text { Bovins } \\
\text { viande }\end{array}$ & Ovins & $\begin{array}{l}\text { Polyc.-élev. } \\
\text { (otex 81) }\end{array}$ & $\begin{array}{c}\text { Toutes } \\
\text { otex }\end{array}$ \\
\hline EBE 1991 F par expl. & 253000 & 273000 & 179000 & 129000 & 149000 & 192000 & 224000 \\
\hline EBE 1996 F par expl. & 205000 & 247000 & 194000 & 153000 & 174000 & 205000 & 227000 \\
\hline $\begin{array}{rr}\text { Ecart 1991-1996 } & \text { F par expl. } \\
\text { en } \%\end{array}$ & $\begin{array}{r}-48000 \\
-19\end{array}$ & $\begin{array}{r}-26000 \\
-9\end{array}$ & $\begin{array}{r}15000 \\
9\end{array}$ & $\begin{array}{r}24000 \\
18\end{array}$ & $\begin{array}{r}25000 \\
17\end{array}$ & $\begin{array}{r}13000 \\
7\end{array}$ & $\begin{array}{r}3000 \\
2\end{array}$ \\
\hline Nombre d'expl. $\%$ du total & 5 & 15 & 21 & 9 & 5 & 10 & 100 \\
\hline $\begin{array}{rr}\text { Aides directes F par expl. } 1991 \\
1996\end{array}$ & $\begin{array}{r}9200 \\
173800\end{array}$ & $\begin{array}{r}14900 \\
164700\end{array}$ & $\begin{array}{l}17500 \\
51000\end{array}$ & $\begin{array}{r}51800 \\
109000\end{array}$ & $\begin{array}{r}81600 \\
125800\end{array}$ & $\begin{array}{r}24300 \\
115900\end{array}$ & $\begin{array}{l}22400 \\
87200\end{array}$ \\
\hline $\begin{array}{|ll|}\text { Total aides directes M F } & 1991 \\
& 1996 \\
\end{array}$ & $\begin{array}{r}267 \\
5035 \\
\end{array}$ & $\begin{array}{r}1202 \\
13296 \\
\end{array}$ & $\begin{array}{l}1895 \\
5514\end{array}$ & $\begin{array}{l}2419 \\
5095\end{array}$ & $\begin{array}{l}2198 \\
3391 \\
\end{array}$ & $\begin{array}{l}1316 \\
6266\end{array}$ & $\begin{array}{l}11823 \\
45953\end{array}$ \\
\hline $\begin{array}{ll}\text { Aides directes/EBE en \% } & 1991 \\
& 1996\end{array}$ & $\begin{array}{r}4 \\
85\end{array}$ & $\begin{array}{r}6 \\
67\end{array}$ & $\begin{array}{l}10 \\
26\end{array}$ & $\begin{array}{l}40 \\
71\end{array}$ & $\begin{array}{l}55 \\
72\end{array}$ & $\begin{array}{l}13 \\
57\end{array}$ & $\begin{array}{l}10 \\
38\end{array}$ \\
\hline
\end{tabular}

* Les exploitations laitières recevraient un peu plus de 5 milliards d'aides directes (soit $12 \%$ du total pour $20 \%$ des agriculteurs). Ces aides ne représenteraient toutefois que $26 \%$ du revenu (contre $10 \%$ en 1991).

* Les éleveurs de viande bovine, ou ovine, seraient destinataires de 8,5 milliards d'aides directes ( $18 \%$ des aides, pour $14 \%$ des agriculteurs). Déjà très dépendants de ces recettes, ces agriculteurs verraient cette dépendance s'accroître d'ici 1996.

* Enfin, pour les exploitations de polycultureélevage, les aides s'élèveraient à 6 milliards et constitueraient plus de la moitié du revenu.

Tous ces calculs sont fondés sur l'Excédent Brut d'Exploitation (EBE) comme critère de revenu. Le poids des aides dans le résultat courant, ou dans le revenu disponible, serait en conséquence très nettement supérieur.

\section{ASPECTS REGIONAUX}

Les cartes ci-après représentent la variation relative de l'EBE moyen de 1991 à 1996 dans les sous-ensembles régionaux relevant d'une même catégorie de handicaps géographiques (montagne, autres zones défavorisées, zones de plaine).

\section{III.1 Les régions de "grandes cultures"}

En grande culture céréalière (OTEX 11), certaines régions présentent une baisse relative de revenu (EBE) moins marquée que celle constatée globalement pour cette orientation. Certains éléments d'explications peuvent être avancés pour rendre compte de cette disparité :

- l'impact du gel des terres est atténué dans certaines régions du fait de la présence de petits productcurs, excmptés de cette mesure ;

- la différence positive entre rendement de référence, assiette des aides, et rendement réel des 
exploitations de l'échantillon varie selon les régions et détermine des compensations plus ou moins élevées des pertes.

L'impact négatif de la réforme est moins marqué dans les orientations d'autres grandes cultures (OTEX 12) qu'en céréaliculture spécialisée (OTEX 11) grâce à la présence de cultures "hors PAC" (betteraves, pommes de terre, ...) qui amortissent les baisses de recettes enregistrées par ces exploitations.

\section{III.2 Les bassins laitiers}

Les effets de la réforme sur les exploitations laitières présentent certaines constantes à travers les régions: compensation assurée par les aides culturales et notamment celles accordées au maïs fourrage, assez faible dispersion de l'amélioration de l'EBE autour d'une moyenne nationale de $9 \%$. Une différenciation régionale se dessine toutefois entre bassins intensifs et bassins herbagers, élevages de plaine et élevages montagnards.

Selon les cas, les compensations proviennent des aides aux cultures, dont le maïs fourrage, ou des aides afférentes aux situations plus extensives : revalorisation des indemnités de handicaps, prime à l'herbe. Les aides spécifiquement accessibles aux exploitations laitières herbagères apparaissent essentielles pour atténuer l'important avantage relatif obtenu par les élevages laitiers bénéficiant des primes au maïs fourrage.

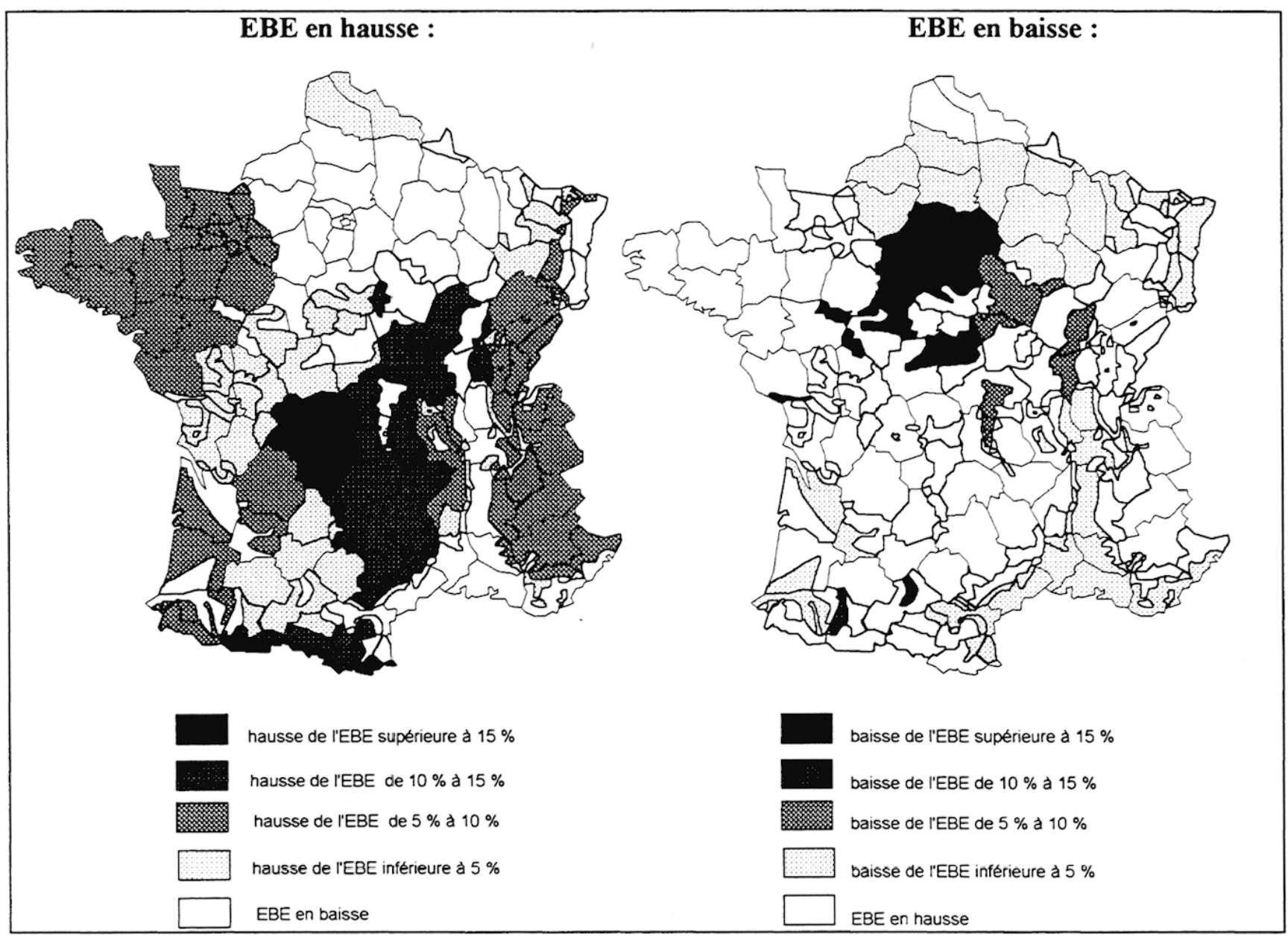

\section{III.3 Les bassins allaitants}

Les éleveurs des grands bassins allaitants traditionnels bénéficient de hausses de revenu comparables (17 à $25 \%$ ), sous l'effet des mécanismes induits par le caractère extensif de ces élevages: surprimes d'extensivité, prime à l'herbe, revalorisation des indemnités de handicaps.

Les avantages relatifs des situations extensives et herbagères ne jouent pas dans les élevages des zones plus favorisées, pratiquant l'engraissement, et, bien que bénéficiaires des aides aux cultures intraconsommées, ceux-ci enregistrent des progressions de revenu moins importantes $(+2 \%$ en Bretagne), sous l'effet des plafonds de chargement.

\section{III.4 Les régions ovines}

Dans toutes les régions où l'OTEX 44 est notablement représentée, cette orientation connaît une forte progression de revenu (14 à 23\%). Dans la population étudiée, ces élevages sont situés surtout dans des zones défavorisées ou d'altitude, d'où la part importante prise dans la compensation 
de leurs pertes par les aides caractéristiques de ces régions: prime à l'herbe, indemnités de handicaps, complément "monde rural" à la prime ovine.

\section{III.5. Incidences régionales du plan d'accompagnement}

Le plan national d'accompagnement est déterminant dans l'augmentation du revenu constatée en Rhône-Alpes, Aquitaine, Midi-Pyrénées et PoitouCharente, où ces mesures représentent plus de $80 \%$ de la progression de l'EBE.
Dans ces régions, où dominent les productions végétales dont les pertes sont sous-compensées, l'EBE aurait progressé plus faiblement, voire régressé (Rhône-Alpes) en l'absence du plan d'accompagnement. En Franche-Comté, où domine la production laitière à l'herbe, le plan d'accompagnement est essentiel à la progression du revenu moyen. Il y contribue pour $80 \%$. En Auvergne et Limousin, ce pourcentage atteint respectivement $60 \%$ et $40 \%$.

Variation relative de l'EBE par région et par OTEX :

\begin{tabular}{|c|c|c|c|c|c|c|c|c|c|}
\hline & \multicolumn{2}{|c|}{ Céréales } & \multicolumn{3}{|c|}{ Bovins } & \multirow{2}{*}{$\begin{array}{c}\text { Ovins } \\
\text { caprins } \\
\text { aut. herbiv. }\end{array}$} & \multirow[t]{2}{*}{ Polyculture } & \multirow{2}{*}{$\begin{array}{c}\text { Grandes } \\
\text { cultures } \\
\text { herbivores }\end{array}$} & \multirow{2}{*}{$\begin{array}{l}\text { Toutes } \\
\text { OTEX }\end{array}$} \\
\hline & Céréales & $\begin{array}{l}\text { aut. gdes } \\
\text { cultures }\end{array}$ & lait & viande & $\begin{array}{c}\text { lait } \\
\text { viande }\end{array}$ & & & & \\
\hline Ile de France & -22 & \begin{tabular}{|l|}
-16 \\
\end{tabular} & & & & & & & -15 \\
\hline Champagne Ardennes & -5 & -12 & 12 & & 14 & & -7 & -12 & -3 \\
\hline Picardie & -4 & -7 & 10 & & & & & 5 & -3 \\
\hline Haute-Normandie & & -12 & 10 & & 6 & & & 3 & -2 \\
\hline Centre & -27 & -11 & 6 & 18 & & 23 & 9 & 11 & -11 \\
\hline Basse-Normandie & & -22 & 8 & 21 & 14 & & & 5 & 6 \\
\hline Bourgogne & -11 & -8 & & 25 & & 22 & & 8 & 5 \\
\hline Nord-Pas-de-Calais & & -2 & 8 & & & & -1 & 9 & 4 \\
\hline Lorraine & & -12 & 8 & & 6 & & & 2 & 1 \\
\hline Alsace & -17 & -6 & 9 & & & & & & -1 \\
\hline Franche-Comté & & -6 & 8 & & 7 & & & 5 & 7 \\
\hline Pays de la Loire & & -18 & 10 & 14 & 13 & 19 & -1 & 9 & 6 \\
\hline Bretagne & & 1 & 9 & 2 & 10 & & & 5 & 6 \\
\hline Poitou-Charentes & -23 & -8 & 8 & 16 & & 17 & -1 & 11 & 2 \\
\hline Aquitaine & -5 & -5 & 8 & & & 11 & 6 & 5 & 2 \\
\hline Midi-Pyrénées & -22 & -6 & 8 & 22 & & 17 & -7 & 11 & 5 \\
\hline Limousin & & & 9 & 17 & 33 & 22 & & & 16 \\
\hline Rhône-Alpes & -26 & -16 & 6 & 18 & 6 & 23 & -2 & -2 & 2 \\
\hline Auvergne & & -17 & 9 & 21 & 21 & 14 & & 9 & 12 \\
\hline Languedoc-Roussillon & & -19 & 11 & 24 & & 21 & -10 & & 1 \\
\hline Provence-Alpes-C. A. & & -24 & & & & 15 & 5 & & 0 \\
\hline Corse & & & & & & 21 & & & 8 \\
\hline Total France & -19 & -9 & 9 & 18 & 12 & 17 & -1 & 7 & 2 \\
\hline
\end{tabular}

\section{LA DISPERSION DES RESULTATS}

L'excédent brut d'exploitation augmenterait de plus de $2 \%$ pour près de 90 des éleveurs, alors que cette progression n'est enregistrée que pour deux tiers des exploitations mixtes ("grandes cultures et herbivores"). A l'opposé $87 \%$ des céréaliers et $61 \%$ des exploitations orientées vers les "céréales et autres grandes cultures" verraient, toutes choses égales par ailleurs, leurs revenus baisser.

Distribution des exploitations (\%) selon la variation relative de l'EBE :

\begin{tabular}{|c|c|c|c|c|c|c|c|c|c|}
\hline & \multicolumn{4}{|c|}{ EBE en baisse } & EBE stable & \multicolumn{3}{|c|}{ EBE en hausse } & Total \\
\hline Orientations (OTEX) & Total & $<-25 \%$ & -25 à $-10 \%$ & -10 à $-2 \%$ & $-2 \grave{a}+2 \%$ & $+2 \grave{a}+10 \%$ & $+10 \mathrm{à}+25 \%$ & $>+25 \%$ & \\
\hline Céréales & 77 & 25 & 35 & 17 & 5 & 6 & 4 & 7 & 17 \\
\hline Céréales et autres grandes cultures & 61 & 10 & 29 & 22 & 12 & 10 & 7 & 9 & 26 \\
\hline Bovins lait & 3 & 0 & 0 & 3 & 10 & 44 & 34 & 10 & 88 \\
\hline Bovins élevage et viande & 5 & 0 & 3 & 2 & 4 & 16 & 40 & 35 & 91 \\
\hline Bovins lait, élevage et viande & 6 & 0 & 0 & 6 & 8 & 27 & 35 & 24 & 86 \\
\hline Ovins, caprins et autres herbivores & 3 & 0 & 0 & 3 & 6 & 17 & 39 & 35 & 91 \\
\hline Polyculture & 38 & 5 & 14 & 19 & 19 & 17 & 10 & 15 & 42 \\
\hline Grandes cultures et herbivores & 19 & 2 & 6 & 11 & 12 & 24 & 22 & 22 & 68 \\
\hline TOUTES ORIENTATIONS & 21 & 4 & 8 & 9 & 21 & 24 & 20 & 14 & 58 \\
\hline
\end{tabular}

L'excédent brut diminuerait de plus de $25 \%$ pour un quart des exploitations céréalières, et cette baisse serait comprise entre 10 et $25 \%$ pour $35 \%$ d'entre elles. Pour les autres exploitations de grandes cultures ces proportions seraient, respectivement, de 10 et $29 \%$. 
Les baisses de revenu seraient donc particulièrement prononcées (plus de $25 \%$ de l'EBE) pour $4 \%$ des exploitations agricoles "professionnelles", soit 20000 entreprises. Parmi celles-ci $75 \%$ sont principalement orientées vers la production de céréales et d'autres "grandes cultures". La nécessité d'adapter rapidement leurs comportements économiques et techniques est donc particulièrement pressante pour ces agriculteurs.

\section{SENSIBILITE DU REVENU A CERTAI- NES HYPOTHESES D'ADAPTATION}

En complément de l'approche précédemment développée il nous a semblé nécessaire de présenter les premiers résultats obtenus en introduisant certaines modifications (de manière exogène) pour les exploitations dont les revenus seraient les plus durement touchés par la réforme.

\section{V.1. Les hypothèses retenues}

Confrontés à une baisse de revenu importante, quelles seront les adaptations mises en oeuvre par les agriculteurs? Les hypothèses sont à l'heure actuelles nombreuses. Pour ne citer que les principales mentionnons : l'agrandissement ; la diminution des investissements grâce à un accroissement de l'utilisation de matériel en commun ; les économies de charges variables ; les augmentations de rendement ; une modification des assolements ; etc... L'outil utilisé pour traduire ces modifications (PECARI) ${ }^{5}$ n'étant pas un "modèle" dynamique les hypothèses utilisables sont, par nature, simplificatrices et partielles. Nous en avons retenu trois, à savoir :

- substitution du blé dur par du blé tendre dans les régions où n'existe plus d'aide spécifique au blé dur (S1) ${ }^{6}$;

- diminution de $10 \%$ des charges variables affectées à la S.C.O.P. ${ }^{7}$, toutes choses égales par ailleurs (S2);

- augmentation de $10 \%$ des rendements en céréales, oléagineux et protéagineux, toutes choses égales par ailleurs (S3).

A cette étape du travail nous avons limité nos investigations aux seules exploitations spécialisées en production céréalière.

\section{V.2. Une marge réelle, mais limitée}

Pour les producteurs spécialisés dans la culture de céréales (OTEX 11) la baisse de revenu s'élèverait à 47800 francs, en moyenne par exploitation. Une baisse de $10 \%$ des charges variables affectées à la SCOP leur assurerait un supplément de revenu de

\footnotetext{
5 Programme d'Evaluation des Conséquences de la réforme A partir du RIca.

6 Substitution effectivement constatée dans les assolement 1993-1994 au nord de la Loire.

${ }^{7}$ Surface en Céréales, Oléagineux et Protéagineux.
}

$14100 \mathrm{~F}$. Par contre une augmentation, dans les mêmes proportions, des rendements leur procurerait un avantage estimé à $27900 \mathrm{~F}$. Enfin, l'abandon du blé dur au profit du blé tendre permettrait d'augmenter le revenu de $7100 \mathrm{~F}$.

Variations de revenu selon différents scénarios Exploitations céréalières (otex 11) - Francs par exploitation :

\begin{tabular}{|l|c|c|r|r|c|}
\cline { 5 - 6 } \multicolumn{2}{c}{} & \multicolumn{3}{c|}{$\begin{array}{c}\text { Supplément de revenu } \\
\text { par scénario }\end{array}$} \\
\hline REGION & $\begin{array}{c}\text { EBE } \\
1991\end{array}$ & $\begin{array}{c}\text { variation } \\
91-96\end{array}$ & S.1 & S.2 & S.3 \\
\hline Ile de France & 370500 & -79300 & 2500 & 20300 & 43100 \\
\hline Champagne-Ard. & 223200 & -4800 &.. & 15300 & 26400 \\
\hline Picardie & 256000 & -8900 &.. & 21400 & 35300 \\
\hline Centre & 344600 & -93400 & 22700 & 17800 & 36500 \\
\hline Bourgogne & 289000 & -31400 &.. & 15900 & 31400 \\
\hline Alsace & 295700 & -49600 &.. & 15100 & 31000 \\
\hline Poitou-Charentes & 200000 & -44700 & 4000 & 12500 & 23600 \\
\hline Aquitaine & 206200 & -9500 &.. & 9000 & 18200 \\
\hline Midi-Pyrénées & 147000 & -27000 &.. & 9400 & 18200 \\
\hline Rhône-Alpes & 160500 & -38100 &.. & 9100 & 16200 \\
\hline France entière & 252900 & -47800 & 7100 & 14100 & 27900 \\
\hline
\end{tabular}

Si l'on admet que les deux dernières hypothèses (S2 et S3) correspondent à une "résorption des inefficacités techniques" (E.Boutitie, D.Vermersch, 1993), il serait donc possible, pour ces exploitations, de réduire notablement leurs pertes de revenu. Ainsi, combinée à la substitution blé dur/blé tendre (déjà réalisée dans les emblavements de la campagne 1992-93), une progression des rendements de $10 \%$ à charges variables inchangées (effets du progrès génétique, par exemple) permettrait de stabiliser la baisse de revenu à environ un quart de celle observée précédemment. Une réduction des charges de $20 \%$ à rendements constants (suppression de certaines interventions phytosanitaires à caractère d'assurance contre le risque, par exemple) serait nécessaire pour parvenir à des résultats similaires.

Les résultats présentés ci-dessous permettent d'estimer les gains réalisables selon diverses autres hypothèses d'évolution des rendements et/ou des charges.

Sensibilité du revenu aux gains de productivité Exploitations céréalières (OTEX 11) Suppléments de revenu en Francs par exploitation :

\begin{tabular}{|c|c|c|c|c|c|c|}
\hline \multirow{2}{*}{$\begin{array}{c}\text { Baisse des } \\
\text { charges } \\
\text { variables de }:\end{array}$} & \multicolumn{6}{|c|}{ Augmentation des rendements de : } \\
\cline { 2 - 7 } & $0 \%$ & $2 \%$ & $4 \%$ & $6 \%$ & $8 \%$ & $10 \%$ \\
\hline $0 \%$ &.. & 5600 & 11200 & 16800 & 22400 & 28000 \\
\hline $2 \%$ & 2800 & 8400 & 14000 & 19600 & 25200 & 30800 \\
\hline $4 \%$ & 5600 & 11200 & 16800 & 22400 & 28000 & 33600 \\
\hline $6 \%$ & 8400 & 14000 & 19600 & 25200 & 30800 & 36400 \\
\hline $8 \%$ & 1200 & 16800 & 22400 & 28000 & 33600 & 39200 \\
\hline $10 \%$ & 14000 & 19600 & 25200 & 30800 & 36400 & 42000 \\
\hline
\end{tabular}

\section{V.3. De fortes différences régionales}

Une augmentation de $10 \%$ des rendements, toutes choses égales par ailleurs, permettrait à quatre régions de compenser intégralement les effets du gel des terres et de la baisse des prix des produits. 
Il s'agit de : Champagne-Ardennes, Picardie, Bourgogne et Aquitaine.

Pour les deux régions les plus touchées (Ile de France et Centre) la croissance de la production représenterait entre $39 \%$ (Centre) et $54 \%$ (Ile de France) des pertes occasionnées par la réforme. En cumulant les effets précédemment mentionnés et un changement d'assolement (substitution du blé dur par du blé tendre) on parvient ainsi à un gain de près de $60000 \mathrm{~F}$. par exploitation dans la région Centre, et de 46000 F. en lle de France. Ce serait donc $60 \%$ des pertes de recettes qui pourraient être compensées dans ces deux régions.

Pour les exploitations les plus performantes, les solutions étudiées ne permettent donc pas de retrouver des niveaux de revenu identiques à ceux obtenus avant la mise en oeuvre de la réforme de la PAC Toutefois ces revenus restant élevés pour les exploitations du Centre et de l'Ile de France, l'accroissement de surface reste une solution financièrement envisageable. Il n'en sera probablement pas de même dans les régions où les revenus étaient déjà modestes.

\section{CONCLUSION}

Les nouveaux dispositifs communautaires ne remettent pas fondamentalement en cause la hiérarchie des revenus.

La substitution -partielle- du soutien des prix par un système de compensations directes rend désor- mais plus transparent le mécanisme des aides à l'agriculture. De plus, l'augmentation de près de 35 milliards de francs des aides directes risque, si les économies réalisées sur les restitutions à l'exportation ne sont pas à la hauteur des résultats escomptés, d'entraîner un accroissement important des dépenses consacrées au secteur agricole. Ceci pourrait réactiver le débat social sur la finalité des aides (effet redistributif, prise en compte de l'environnement et de l'aménagement de l'espace, ...).

Le semi-découplage entre les aides directes servies et les niveaux individuels de production a pour effet d'octroyer de meilleures compensations aux exploitations dont les productivités apparentes (de l'hectare ou du cheptel) les plus faibles. Ce mécanisme ne condamne pas nécessairement la recherche, sous certaines formes, de gains de productivité.

Un autre aspect "dynamique", non pris en compte dans ces simulations, est celui des concurrences inter régionales qui pourront être induites par la réforme pour certaines productions : ainsi, l'avantage immédiat dont bénéficient les formes "extensives" de production de viande pourrait être remis en cause par la baisse du coût de l'alimentation dans les productions intensives ou hors-sol, de même, des spécialisations régionales non directement concernées par la réforme (cultures légumières) pourraient pâtir des adaptations d'assolement de certaines régions céréalières.

\section{REFERENCES BIBLIOGRAPHIQUES}

E. BOUTTIIE, D. VERMERSCH (1993) - L'évolution des rendements céréaliers : les difficultés de la maîtrise de l'offre. INRA-Sciences Sociales, $\mathrm{n}^{\circ} 1$ - janvier.

J.P. BUTAULT, B. DESBROSSES, WAVRESKY (1993) Situation économique des éleveurs bovins et réforme de la PAC. SFER - session de printemps. 13-14 mai.

R. CARLES, CHITRT, G. MILLET (1993) - Marges et coût de production du blé tendre et de quelques autres produits de grande culture. Estimation des effets de la nouvelle PAC. SFER - session de printemps. 13-14 mai .

F. COLSON, D. ESARMENIEN (1993) - La réforme de la PAC et les producteurs de viande bovine : effets sur la répartition des aides publiques, conséquences sur les revenus, stratégies d'adaptation possibles. SFER - session de printemps. 13-14 mai.

M. DESRIERS, B. PERREL, J.P. STRAUSS (1993) - Des aides plus favorables à l'élevage qu'aux grandes cultures. AGRESTE Cahiers, $n^{\circ} 14$, - juin

INSEE (1992) - La mutation de l'agriculture. Economie et Statistique, $n^{\circ}$ 254-255 - mai-juin

J. LOYAT (1993) - Intervention publique, revenu agricole et efficacité (modèle EKAM). SFER - session de printemps. 13-14 mai

J. RAMANANTSOA, E. BOUTTIIE, M.A. MATHIEU (1993) - Evaluation des revenus agricoles (modèle MAGALI. SFER - session de printemps. 13-14 mai . 